\title{
Atrial fibrillation precipitated by tyramine containing foods
}

\author{
LEONARD H JACOB, D B CARRON \\ From the Grimsby District General Hospital, Grimsby, South Humberside
}

SUMMARY Episodes of atrial fibrillation that occurred after meals developed in a 60 year old man with a history of ischaemic heart disease. The attacks were precipitated by precursors and metabolites of tyramine and tyramine containing foods and drinks, in the absence of monoamine oxidase inhibitors. The patient has remained free of atrial fibrillation for the past twelve months on a diet that does not contain tyramine.

\section{Case report}

In 1980 , at the age of 56 years, our patient was admitted to the hospital with central chest pain radiating to the left arm and in fast atrial fibrillation. Electrocardiography showed $Q$ waves and ST segment elevation in the anterior chest leads V1-V5, and the plasma activity of the cardiac enzymes was increased, confirming the diagnosis of acute anterior myocardial infarction with atrial fibrillation. A few hours later acute left ventricular failure developed. He was treated with digoxin, diuretics, and anticoagulants. He quickly improved and recovered and sinus rhythm returned. He was transferred from the coronary care unit to the medical ward where he convalesced for 10 days and made a good recovery. Investigations-that is full blood count, urea and electrolyte concentration, liver function and thyroid function tests-were normal. He was discharged home in sinus rhythm on the above drugs.

He had a history of migraine in his twenties, which had been successfully treated by his general practitioner. There was no history of palpitation or heart disease nor of any psychiatric illness. He was married with three children and was a non-smoker. He drank two pints of beer a night and was not on any drugs. His father had died of heart disease at the age of séventy but there was no other relevant family history.

Two months later when he was reviewed in the outpatient clinic his condition was good, he was in sinus rhythm, and he was not in heart failure. He was maintained on digoxin and the other drugs were

Requests for reprints to Dr D B Carron, Grimsby District General Hospital, Scartho Road, Grimsby, South Humberside DN33 2BA. stopped. He was subsequently reviewed throughout 1981 at the clinic and his condition remained stable; he was eventually discharged back to the care of his general practitioner.

In February 1983 he was referred to the hospital after he developed attacks of palpitation and dyspnoea, and clinical examination disclosed atrial fibrillation and left ventricular failure, which were confirmed by electrocardiogram and chest $x$ ray respectively. The dose of digoxin was increased to $250 \mu \mathrm{g} /$ day and diuretics were started. He improved and was subsequently reviewed in the clinic and found to be in a stable condition and in sinus rhythm with a heart rate of 64 beats $/ \mathrm{min}$; the attacks of palpitation had become less frequent.

In May 1983 during one of his clinic reviews he mentioned for the first time that some of the attacks of palpitation occurred after meals. When he was questioned more closely about foods he suspected he identified cheese, chocolate, red wine, and bananas. Later after he had been asked to note his food more closely, he mentioned that broad beans and some tinned food with preservatives had also precipitated palpitation. Urinary concentrations of catecholamines and 5-hydroxyindolacetic acid were normal, however.

There was clearly some connection between the foods he mentioned and the palpitation which was not prevented by digoxin. A change to amiodarone improved control but did not abolish palpitation. $\mathrm{He}$ was advised to avoid foods containing tyramine, phenylalanine, and tyrosine. Two 24 hour Holter recordings obtained while he was on this diet showed sinus rhythm and no episodes of atrial fibrillation. Atrial fibrillation occurred when he was challenged with tyramine containing foods during 24 hour Hol- 
ter recording; this coincided with the occurrence of palpitation. Palpitation occurred about one hour after the challenge, despite continuing amiodarone treatment, and resolved when sinus rhythm returned three to four hours after meals.

Urine collected during the test contained normal concentrations of catecholamines. A tyramine provocative test was planned, but a right sided transient ischaemic attack developed after a bout of palpitation brought on by his eating home baked bread made with dried yeast. This episode was considered to be embolic and he was started on anticoagulants. He later declined the provocation test.

Repeated outpatient reviews in 1985 showed that he remained in regular sinus rhythm without any attacks of palpitation. He was on amiodarone $200 \mathrm{mg}$ per day and warfarin according to the British Comparative Ratio.

After he had been in sinus rhythm for more than six months he agreed to be challenged. Holter recording for 24 hours again disclosed a period of atrial fibrillation that coincided with reports of palpitation in the patient's symptoms diary and occurred after consumption of the specially prepared high tyramine test diet. Sinus rhythm returned within a few hours.

For the past 12 months he has taken a diet free of tyramine, its precursors, and metabolites, and has not had palpitation. He was last reviewed in the outpatient clinic in March 1986 when he was in sinus rhythm and in good health.

\section{Discussion}

Foods and drinks such as cheese, dried yeast, red wine (Chianti), yoghurt, pineapple, bananas, ice cream, and chocolate are known to produce hypertensive crises in patients on monoamine oxidase inhibitors. Phenylalanine, tyramine, serotinin, tyrosine, and noradrenaline are the vasoactive amines most commonly found in these foods. ${ }^{1}$ Phenylalanine is metabolised to tyrosine, which is decarboxylated to tyramine and also to dopa, dopamine, and noradrenaline. ${ }^{2}$ Tyramine increased nor- $\stackrel{c}{\stackrel{c}{.}}$ adrenaline in the coronary sinus blood in patients $\overrightarrow{\vec{s}}$ with ischaemic heart disease. ${ }^{3}$ The increase in the heart rate-blood pressure product was not, however, $\frac{C}{0}$ found to be significant. Cardiac arrhythmias, such as sinus tachycardia, atrial ectopic impulses, ventricular ectopic impulses, and Wenckebach's phenomenon were induced in patients with pharma- os cologically untreated anxiety and depression when $\vec{O}$ they were challenged with tyramine infusion ${ }^{4}$ but to our knowledge atrial fibrillation has not been ${ }_{0}^{\omega}$ reported.

Although in our patient acute myocardial of infarction was complicated initially by atrial iv fibillation, sinus rhythm returned while he was in $\tilde{N}$ hospital after the infarction and continued after dis- or charge. Palpitation developed after consumption of $\bigcirc$ tyramine containing foods and he was not on anti- depressant drugs such as monoamine oxidase Ti inhibitors. On two separate occasions tyramine chal- : lenge produced atrial fibrillation that was confirmed on 24 hour Holter recordings. We think that tyramine containing foods can precipitate cardiac $\vec{\emptyset}$ arrhythmias, such as atrial fibrillation, in some $\stackrel{\infty}{-}$ patients with ischaemic heart disease.

We thank Miss A Priestley for the specialised dietetic advice.

\section{References}

1 Lovenberg DW. Psycho and vaso-active compounds in food substances. I Agric Food Chem 1974;22:23-6.

2 Martin DW, Mayes PA, Rodwell VW, Granner DK. Harper's review of biochemistry. 20th ed. California: Lang, 1985: 280, 305, 306, 327.

3 Forman MB, Robertson D, Goldberg M, et al. Effect of tyramine on myocardial catecholamine release in $\delta$ coronary heart disease. $\mathrm{Am} \mathcal{F}$ Cardiol 1984;53:476-8P.

4 Tiller JWG, Dowling JT, Tung LH, Maguire KP, Rand MJ, Davies BM. Tyramine induced cardiac $\frac{\mathrm{O}}{\mathrm{O}}$ arrhythmias. N Engl f Med 1985;313:266-7. 